\title{
Die neuen medizinisch-ethischen Richtlinien der SAMW zur Betreuung von Patientinnen und Patienten am Lebensende
}

\author{
M. Salathé, M. Zimmermann-Acklin
}

Die Veröffentlichung der Richtlinien zur «Betreuung von Patienten am Lebensende» zur Vernehmlassung im Februar 2004 löste ein grosses Echo aus. Sowohl in der Medienberichterstattung als auch in den über 150 eingegangenen Stellungnahmen stand die Regelung der ärztlichen Beihilfe zum Suizid im Vordergrund. Von den kantonalen Ärztegesellschaften, den medizinischen Fachgesellschaften und kantonalen Behörden inklusive Kantonsärzten wurde die bedingte Öffnung im Bereich der ärztlichen Beihilfe zum Suizid mehrheitlich begrüsst. Kritisch zur Neuregelung äusserten sich vor allem einzelne Ärztinnen und Ärzte, andere Einzelpersonen sowie religiöse Kreise.

Ein weiterer Schwerpunkt der Vernehmlassungsantworten bezog sich auf die Regelung der Entscheidfindung bei nicht urteilsfähigen Patientinnen und Patienten, welche in der nun verabschiedeten Fassung der Richtlinien zwar inhaltlich unverändert, jedoch klarer strukturiert vorliegt.

Zur ärztlichen Beihilfe zum Suizid hatte die SAMW in den «Medizinisch-ethischen Richtlinien für die ärztliche Betreuung sterbender und zerebral schwerst geschädigter Patienten» von 1995 ausdrücklich festgehalten, dass diese kein Teil der ärztlichen Tätigkeit sei. Die nun vom Senat verabschiedeten neuen «Richtlinien zur Betreuung von Patientinnen und Patienten am Lebensende», welche zerebral schwerstgeschädigte Langzeitpatienten nicht mehr mit einschliessen, halten an dieser Aussage fest, tragen aber gleichzeitig dem gewandelten Verständnis der Patientenautonomie Rechnung, indem sie die Suizidbeihilfe insgesamt offener regeln. Diese Neuregelung soll dazu beitragen, die strafrechtlich nicht verbotene Praxis der Suizidbeihilfe - es geht momentan gemäss EURELD-Studie um 0,36\% aller Todesfälle in der Schweiz - auch für Ärztinnen und Ärzte durch klare Leitlinien zu regeln.

Die Richtlinien stellen in aller Deutlichkeit

Korrespondenz:

lic. iur. Michelle Salathé SAMW

Petersplatz 13

CH-4051 Basel

E-Mail: m.salathe@samw.ch fest, dass die Rolle des Arztes bei Patientinnen und Patienten am Lebensende darin besteht, «Symptome zu lindern und den Patienten zu begleiten». Aufgrund der Vernehmlassungsergebnisse wurde an dieser Stelle noch ergänzend hinzuge- fügt: «Es ist nicht seine Aufgabe, von sich aus Suizidhilfe anzubieten, sondern er ist im Gegenteil dazu verpflichtet, allfällige Suizidwünschen zugrundeliegende Leiden nach Möglichkeit zu lindern.»

Allerdings könne der Arzt, so halten die Richtlinien in der zur Vernehmlassung veröffentlichten Fassung fest, in einzelnen Fällen in eine Konfliktsituation geraten, die dann eine Gewissensentscheidung erforderlich macht. Es heisst darum: «Auf der einen Seite ist die Beihilfe zum Suizid nicht Teil der ärztlichen Tätigkeit, weil sie den Zielen der Medizin widerspricht. Auf der anderen Seite ist die Achtung des Patientenwillens grundlegend für die Arzt-Patienten-Beziehung. Diese Dilemmasituation erfordert eine persönliche Gewissensentscheidung des Arztes. Die Entscheidung, im Einzelfall Beihilfe zum Suizid zu leisten, ist als solche zu respektieren.»Anschliessend werden die Mindestanforderungen erwähnt, die im Falle einer Entscheidung zur Beihilfe einzuhalten sind.

Den in der Vernehmlassung mehrfach geäusserten Bedenken, der Patient könne unter Umständen Druck auf seinen Arzt ausüben, Beihilfe zum Suizid zu leisten, wurde in der definitiven Fassung Rechnung getragen. Neu wird ausdrücklich festgehalten, dass ein Arzt in jedem Fall das Recht hat, die Suizidbeihilfe abzulehnen. Explizit wurde auch ein Hinweis aufgenommen, wonach Vorgesetzte ihren Mitarbeiterinnen und Mitarbeitern die Beihilfe zum Suizid verbieten, diese oder die Mitwirkung dazu aber nicht von ihnen verlangen können. Trotz verschiedener Anregungen, den Geltungsbereich der Richtlinien auch auf Patientinnen und Patienten ausserhalb der unmittelbaren Sterbephase auszudehnen, wurde an der ursprünglichen engen Fassung festgehalten. Damit bleibt deutlich unterstrichen, dass sich die vorliegende Regelung nicht auf sterbewillige Patientinnen und Patienten bezieht, die an einer schweren Krankheit oder Behinderung leiden.

Eine umfassende palliative Betreuung kann bei diesen Patienten wie auch bei den Patienten am Lebensende dazu beitragen, allfällige Suizidwünschen zugrundeliegende Leiden zu lindern. 